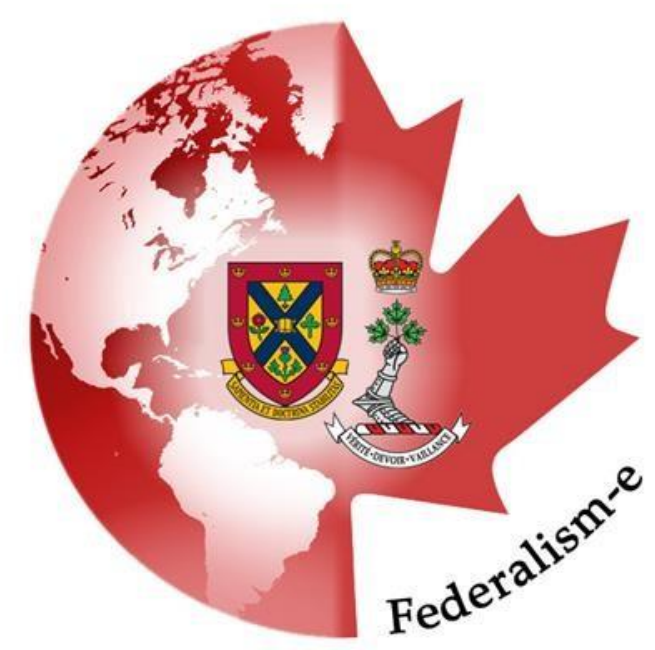

\title{
Transferts fédéraux : la péréquation décortiquée
}

Patrick Mercier, Université de Montréal

Les questions d'ordre économique jouent un rôle important dans les fédérations. C'est d'ailleurs l'un des facteurs qui ont amené des entités différentes à s'unir. La prospérité promise dans l'union suffisait généralement à convaincre les plus récalcitrants de s'y joindre. Ce facteur fut d'ailleurs important dans l'ajout de nouvelles provinces au Canada entre 1867 et 1949. Cependant, au fil des années, des disparités économiques sont apparues entre les provinces. C'est pour pallier ce type de problème qu'existe la péréquation, un sujet dont plusieurs ont déjà entendu parler, mais peu savent en quoi il consiste réellement.

Cet article vise à expliquer différents aspects de la péréquation. Tout d'abord, une courte description de la péréquation sera présentée ainsi que le contexte dans lequel elle se situe à l'égard des transferts fédéraux. Ensuite, une présentation historique depuis sa première application en 1957 suivra. Également, les différents changements survenus dans les dernières années ainsi que la formule de calcul seront décortiqués. Une présentation des difficultés quant à son efficacité et à son application suivra. Pour terminer, de brefs exemples de complications politiques que peut entraîner la péréquation entre les provinces et entre les paliers de gouvernements seront abordés. 


\section{Mise en contexte}

Chaque année fiscale, le gouvernement fédéral octroie des montants aux provinces qui proviennent à même de ses revenus. Ces transferts sont composés de trois principales enveloppes. La plus importante est celle du Transfert canadien en matière de santé (TCS) qui est la contribution du fédéral en matière de santé au Canada. Une autre enveloppe est celle du Transfert canadien en matière de programmes sociaux (TCPS). Finalement, il y a la péréquation. Cette dernière se démarque des deux premières sur plusieurs aspects. Tout d'abord, il s'agit d'un transfert inconditionnel. Le TCS et le TCPS sont assujettis à des conditions de base, mais la péréquation n'est rattachée à aucune condition. De plus, le montant attribué à l'intérieur de ces deux enveloppes est calculé différemment de celui de la péréquation. Depuis l'exercice financier de 2014-2015, les deux transferts conditionnels sont versés dans toutes les provinces selon un montant égal par habitant. Le calcul de la péréquation est beaucoup plus complexe et sera examiné plus loin. Ces types de transferts sont essentiels au bon fonctionnement d'une fédération comme le Canada.

Pour l'année fiscale 2014-2015, le total des trois enveloppes octroyées a représenté 61364 millions de dollars (voir annexe 1-tableau-1 p. 22). De cette somme, le Québec a reçu 19614 millions (voir annexe 1-tableau-2 p 23). Cela représente $31 \%$ du total de ces trois transferts, mais le Québec ne comprend que $23 \%$ de la population canadienne. Comme le TCS et le TCPS sont donnés au prorata de la population, l'écart résulte de l'enveloppe de péréquation.

Dans une fédération idéale, chaque palier de gouvernement devrait avoir accès au revenu dont il a besoin afin de remplir ses responsabilités, ce qui est considéré comme un équilibre vertical. L'inégalité des ressources entre mêmes niveaux de gouvernement est vue comme un déséquilibre horizontal. La fédération idéale n'existant pas, c'est pour cette raison que la majorité des fédérations utilise des transferts tels que la péréquation. L’objectif général de la péréquation est de réduire le déséquilibre horizontal, c'est-à-dire le déséquilibre entre les provinces. La Loi constitutionnelle de 1982 stipule que le Parlement et le gouvernement du Canada : " prennent l'engagement de principe de faire des paiements de péréquation propres à donner aux gouvernements provinciaux des revenus suffisants pour les mettre en mesure d'assurer les services publics à un niveau de qualité et de 
fiscalité sensiblement comparables ". Il est important de bien saisir que la péréquation est payée par le gouvernement fédéral à même les recettes qu'il engrange, ce n'est pas un transfert d'argent d'une province à une autre. Si le principe global est toujours demeuré le même, les changements apportés au fonctionnement au fil du temps ont été nombreux.

\section{Historique}

La péréquation a été établie pour la première fois en 1957. Son implantation faisait suite au rapport de la Commission royale Rowell-Sirois demandé par le fédéral et qui visait à tenter de trouver une solution pour sortir de l'endettement l'Alberta et la Saskatchewan. $\mathrm{Au}$ début, les paiements étaient calculés seulement en fonction des recettes de trois types d'impôts : des particuliers, des entreprises et des droits de succession. Pour savoir combien une province devait recevoir des paiements de transfert, il fallait faire la moyenne des revenus que soutiraient les deux provinces les plus riches (Ontario et ColombieBritannique) des trois types d'impôts et la diviser par la population respective. Ensuite, le fédéral donnait le montant d'argent aux autres provinces pour qu'elles atteignent la moyenne calculée par habitant. Seulement l'Ontario n'en bénéficiait pas. Il avait été décidé que la formule de calcul des paiements pouvait être revue tous les cinq ans.

En 1962, Ottawa décide de prendre en considération une portion des revenus issue des ressources naturelles pour diminuer le nombre de provinces bénéficiaires. Avec cette nouvelle formule, l'Alberta et la Colombie-Britannique cessèrent rapidement de recevoir des paiements. L’Alberta n'était pas satisfaite puisqu'elle arguait que les ressources naturelles relevaient du provincial et qu'elles étaient non-renouvelables, donc que la durée des revenus était indéterminée. Pour que la formule soit considérée comme étant plus égale pour tous et qu'elle réponde au grief de l'Alberta, le nouveau calcul de 1967 prit en compte 16 différents types de revenus. Ce nombre ne cessa de croître lors de futures révisions et au début des années 80, 38 types de revenus étaient pris en considération.

Afin de savoir si une province avait le droit ou non de recevoir des paiements, il fallait savoir si son revenu pour chacune des 38 assiettes fiscales était positif ou négatif comparativement à la moyenne canadienne pour chacun des éléments. Si une province tombait sous les standards, elle était considérée comme ayant un droit positif à la péréquation. Ensuite, il fallait additionner les résultats positifs et négatifs et seulement si 
le calcul final était positif, la province recevait des paiements. Les calculs se faisaient au prorata de la population et en considérant un éventuel taux de taxation équivalent à la moyenne canadienne. Ainsi, une province ne recevait pas moins de péréquation parce que ses revenus issus d'un faible taux de taxation étaient plus faibles que dans une autre province et vice-versa. Ceci est toujours vrai. De plus, il est important de rappeler que les provinces qui terminent le calcul avec un droit négatif n’ont pas à payer de péréquation, puisque c'est le fédéral qui paie à même ses revenus.

Les coûts du programme pour le fédéral ont significativement augmenté à partir de 1973 en raison de la hausse importante du prix des ressources naturelles et plus particulièrement du pétrole. De plus, les provinces percevaient une part de plus en plus importante des revenus issus de l'impôt. En 1977-78, les prix du pétrole avaient atteint un tel niveau que les revenus de l'Alberta tiraient la moyenne canadienne disproportionnellement vers le haut. L’Ontario devenait ainsi une province bénéficiaire, mais le poids fiscal d'une telle mesure n'était pas possible pour le fédéral. C'est ainsi qu'en 1977, seulement 50\% des revenus du pétrole sont pris en compte et que les provinces dont le revenu par habitant est supérieur à la moyenne canadienne ne reçoivent pas de péréquation. De plus, c'est maintenant la moyenne des dix provinces qui est prise en compte. Cette mesure empêche l'Ontario d'en recevoir.

Stevenson (2004) remarque une certaine ironie dans cette situation. En effet, c'était le gouvernement fédéral qui avait décidé en 1962 d'inclure les ressources dans le calcul de la péréquation pour diminuer le nombre de provinces récipiendaires. Toutefois, 15 ans plus tard c'est cette décision qui fait fortement augmenter les coûts des transferts et qui amène Ottawa à ne seulement considérer que $50 \%$ des revenus issus des ressources. Cette décision arbitraire du gouvernement vise à priver l'Ontario de la péréquation.

En 1981-1982, une nouvelle formule de calcul voit le jour. La moyenne nationale ne sera plus calculée en tenant compte des dix provinces, mais seulement de cinq. L'Alberta est exclue du calcul ainsi que les provinces maritimes. C'est aussi en 1982, que la péréquation est enchâssée dans la Constitution canadienne, et ce sans susciter d'importantes réactions puisque le programme existait déjà depuis longtemps et qu'il faisait relativement l'unanimité (Stevenson, 2004, p. 143). Il est important de réaliser que la Constitution indique seulement l'obligation du fédéral à maintenir une égalité relative 
entre les provinces. Les modalités de fonctionnement ne sont pas indiquées et c'est ce qui permet au gouvernement fédéral de changer ou d'adapter les règles comptables au gré des circonstances.

De 1982 à 2004, le programme restera sensiblement le même, mais la formule comptable se complexifie. De nouvelles taxes ont été ajoutées, ainsi qu'un plancher et un plafond. Le fédéral changeait les règles quelque peu, mais l'essentiel demeurait. Au milieu des années 80, le gouvernement central a signé des accords avec Terre-Neuve et la Nouvelle-Écosse concernant leurs ressources naturelles qui se trouvent au large de leurs côtes respectives, ces dernières n'étant pas prises en considération dans le calcul de la péréquation.

À cette époque, pour calculer la péréquation, il fallait d'abord connaître la capacité fiscale de chacune des provinces. Pour ce faire, Ottawa utilisait le régime fiscal représentatif $(\mathrm{RFR})$. Cet indice mesurait le montant que chaque province pouvait retirer des 33 différentes sources de taxes. Ces montants étaient ensuite additionnés et comparés à la moyenne de cinq provinces: Québec, Ontario, Manitoba, Saskatchewan et ColombieBritannique. Les provinces qui se trouvaient sous la moyenne recevaient alors un montant par habitant qui le ramenait à la moyenne. Il est important de noter que ces paiements faits par Ottawa ne rendent pas une véritable égalité interprovinciale. Les provinces les plus pauvres augmentent leur standard, mais les plus riches n'abaissent pas le leur.

Le graphique suivant indique les résultats du calcul pour l'année financière 20032004 et démontre concrètement l'explication précédente. La ligne pointillée rouge représente la moyenne de la norme nationale calculée selon les cinq provinces précédemment énumérées. On constate que trois provinces ne reçoivent pas de péréquation (pratiquement quatre). La zone en brun clair représente le paiement que les provinces bénéficiaires reçoivent pour s'élever à la moyenne nationale de capacité fiscale de 5,916\$ par habitant. En contrepartie, la Saskatchewan, l'Ontario et l'Alberta ne voient pas leur capacité fiscale être rabaissée à la moyenne. 


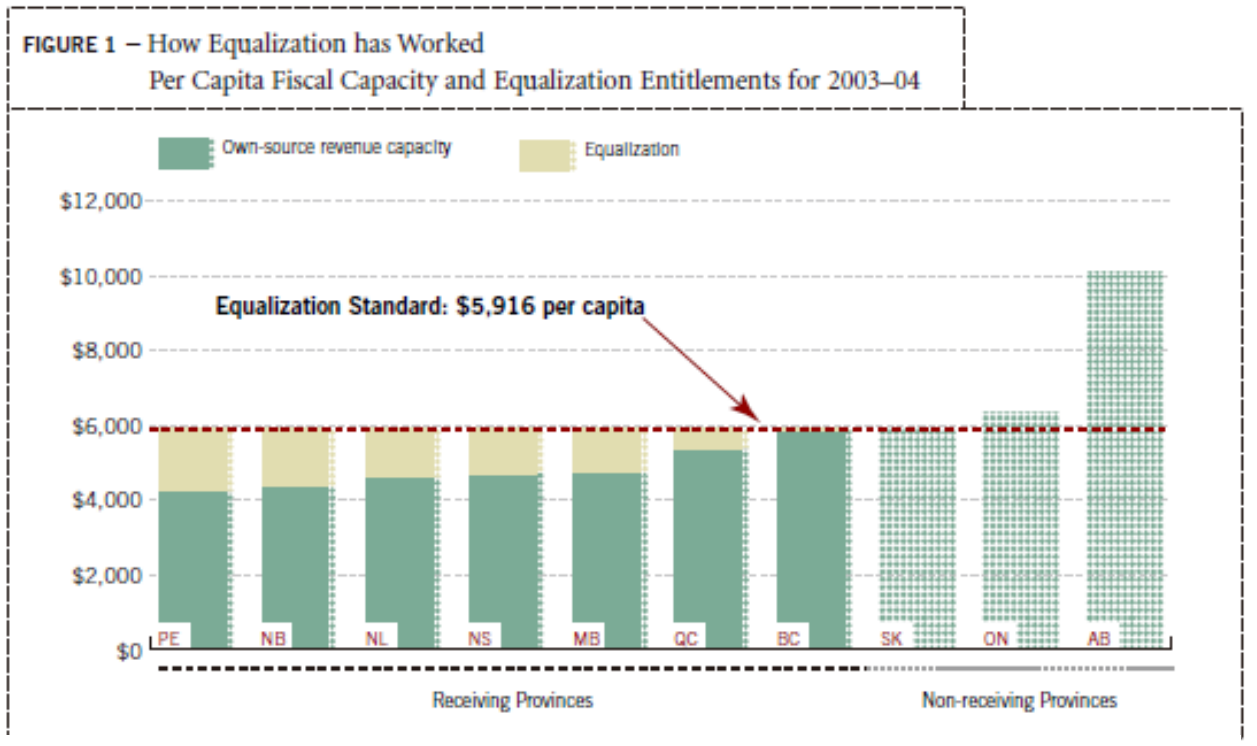

Source : http://publications.gc.ca/Collection/F2-176-2006E.pdf

\section{La péréquation actuellement}

De 2004 à 2007, la formule avait été changée pour une enveloppe fixe dont le montant évoluait chaque année. En 2007, il a été décidé de revenir à un modèle qui ressemblait plus à celui d'avant 2004 et qui a été expliqué plus haut, mais en y faisant tout de même quelques modifications. Les 33 assiettes fiscales ont été regroupées en cinq catégories: impôt sur le revenu des particuliers, impôt sur le revenu des sociétés, taxe de vente, impôts fonciers et $50 \%$ des ressources naturelles. Comme mentionné préalablement, les ressources naturelles n'ont pas toujours été considérées dans le calcul. Leur pourcentage de prise en considération a changé huit fois depuis 1957, variant de $0 \%$ à $100 \%$. Ces variations démontrent un manque de consensus au sein du Canada relativement à l'importance que l'on doit leur accorder dans la formule, puisque les conséquences sont majeures sur les résultats (Bernard, 2012).

Une autre mesure apportée est la mise en place d'un plafond de capacité fiscale (PCF). Cette nouvelle mesure vise à être équitable envers les provinces qui ne reçoivent pas de péréquation. En effet, en n’incluant que 50 \% des ressources naturelles dans le calcul, une province récipiendaire, après avoir reçu la péréquation, pourrait voir son assiette fiscale être plus importante qu'une province qui ne reçoit pas de paiement. Le PCF calcule 
la capacité fiscale de chaque province après péréquation incluant la totalité des revenus tirés des ressources naturelles. Ce nouveau chiffre calculé par province est ensuite comparé à la norme nationale et une province ne peut pas dépasser cette moyenne avec un paiement de péréquation.

Le cas de Terre-Neuve explique bien les conséquences du PCF. En effet, en n’incluant que $50 \%$ de ses ressources, la province devrait être bénéficiaire. Si tel était le cas, sa capacité fiscale deviendrait plus élevée que les provinces qui ne reçoivent pas de paiements, puisqu'il ne s'agit pas de son taux réel. Une fois calculé, le PCF de la province, celle-ci se retrouve au-dessus de la moyenne nationale et ne peut donc pas recevoir de péréquation. Ce calcul abaisse aussi le montant auquel a droit le Québec, le Manitoba et la Nouvelle-Écosse, puisque ces trois provinces ont des revenus issus de ressources naturelles.

Une autre règle a vu le jour en 2009-2010, il s'agit du plafonnement de la croissance de l'enveloppe globale. Ce nouvel élément minimise les risques pour le gouvernement fédéral de voir l'enveloppe globale de péréquation prendre des proportions trop importantes. Ainsi, la croissance du paiement total ne doit pas excéder la moyenne mobile de la croissance du produit intérieur brut nominal des trois années précédentes. Cette règle a eu un impact lors de l'exercice financier 2014-2015. En effet, après avoir calculé la péréquation et appliqué le PCF, l'enveloppe globale aurait dû être de 17,9 milliards de dollars, mais le facteur de croissance était de 3,5\%, ce qui a rabaissé le montant à 16,7 milliards. Cela s'est traduit par une diminution de $50 \$$ par habitant par province. En 2014-2015, six provinces (l'Île-du-Prince-Édouard, la Nouvelle-Écosse, le Nouveau-Brunswick, le Québec, l’Ontario et le Manitoba) se sont partagées les 16,7 milliards de péréquation. De cette somme, le Québec a obtenu 9,3 milliards, soit 55,6\%. La partie la plus importante a été reçue par le Québec, mais celle-ci se classe au cinquième rang sur six lorsque l'on fait le calcul par habitant comme le démontre le tableau suivant. 


\section{April 14, 2017 FEDERALISM-E}

\begin{tabular}{|c|c|c|c|}
\hline & 2013-2014 & 2014-2015 & Variation en $\%$ \\
\hline T.-N.-L. & 0,0 & 0,0 & - \\
\hline Î.-P.-É. & 2339,3 & 2477,6 & $5,9 \%$ \\
\hline N.-É. & 1549,2 & 1728,1 & $11,5 \%$ \\
\hline N.-B. & 2001,4 & 2205,8 & $10,2 \%$ \\
\hline Qc & 961,5 & 1129,7 & $17,5 \%$ \\
\hline Ont. & 234,4 & 145,7 & $-37,8 \%$ \\
\hline Man. & 1418,2 & 1367,4 & $-3,6 \%$ \\
\hline Sask. & 0,0 & 0,0 & - \\
\hline Alb. & 0,0 & 0,0 & - \\
\hline C.-B. & 0,0 & 0,0 & - \\
\hline
\end{tabular}

Il est intéressant de noter que toutes les provinces ont un jour ou l'autre reçu de la péréquation (Alberta 1957-1963). Aussi, l’Ontario reçoit de la péréquation seulement depuis 2009, mais les montants reçus totalisent déjà plus d'argent que ce que l'île-du-PrinceÉdouard et la Saskatchewan ont obtenu dans leur histoire respective (Di Matteo, 2015). Cela démontre qu'il peut être audacieux pour une province de se plaindre lorsqu'elle ne reçoit pas de péréquation parce que la formule l'a désavantagée.

Pendant une longue période de temps, il parut improbable que l'Ontario devienne récipiendaire, mais la crise de 2008 a frappé son secteur manufacturier de plein fouet et les choses ont rapidement changé. Qui plus est, son industrie automobile a dû être sauvée de la faillite par Ottawa. La situation opposée peut aussi s'avérer possible. Terre-Neuve et la Saskatchewan dans une moindre mesure, ont longtemps reçu des paiements, mais aujourd'hui elles font partie des provinces qui ont une assiette fiscale supérieure à la moyenne. L'Institut Fraser présente d'ailleurs un graphique qui symbolise l'évolution du partage de la péréquation à trois époques différentes, représentées par les années 1957, 1990 et 2014. Il est intéressant de remarquer que le Québec accapare une portion de plus en plus importante de l'enveloppe globale, malgré un poids démographique de plus en plus faible sur l'ensemble du Canada (28,8 \% en 1961 contre $23 \%$ en 2015). 


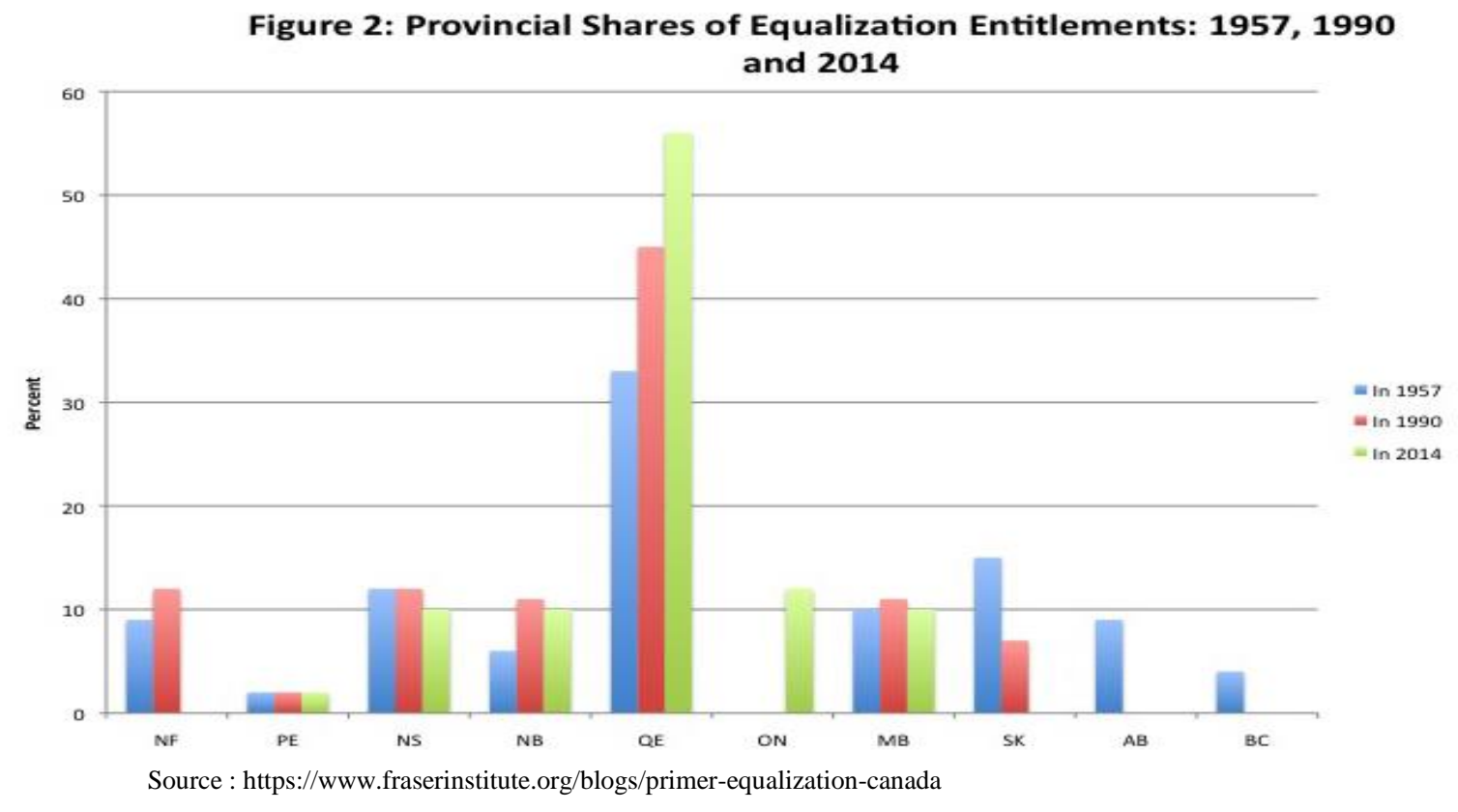

Problèmes reliés à la mise en application

L'historique dressé précédemment, avec tous les changements que cela comporte, ainsi que la complexité des éléments qui entrent dans la formule de calcul ont montré que le programme de péréquation n'a jamais été un élément du fédéralisme facile à comprendre. Toutes les modifications apportées au fil du temps n'ont pas permis de trouver une formule qui serait dénuée de défauts. Dans un document intitulé Le programme canadien de péréquation: fondements, réalisations et défis, l'auteur Jean-Thomas Bernard fait d'ailleurs ressortir quatre problèmes de la mise en application de la péréquation.

Tout d'abord, il y a des problèmes qui sont reliés à la stabilité des paiements : « Les provinces bénéficiaires désirent recevoir des transferts qui sont stables dans le temps, parce que les besoins de leur population sont plutôt stables". En effet, les provinces ne veulent pas avoir à diminuer les services parce qu'elles reçoivent moins d'argent. Les ressources naturelles sont un facteur qui influence beaucoup le montant de l'enveloppe globale, mais celles-ci peuvent subir d'importantes fluctuations de prix sur une courte période de temps et ainsi affecter toutes les provinces. Aussi, les ressources fiscales de chacun des paliers de gouvernement suivent les cycles économiques. Ainsi, si Ottawa fait des paiements stables, même si ses revenus baissent, il se retrouve rapidement avec un manque à gagner. C'est 
d'ailleurs pour éviter ce problème que la hausse de l'enveloppe globale suit la moyenne de l'augmentation du PIB nominal tel qu'expliqué précédemment. Par contre, cela se traduit par un transfert de l'instabilité potentielle envers les provinces. Par exemple, si les revenus fiscaux du Québec venaient à chuter, le fédéral serait limité sur l'augmentation maximum transférable à Québec à cause des limites de l'enveloppe globale. Il faut aussi prendre en compte que lorsqu'une telle limite est atteinte ce qu'une province gagne, une autre le perd, il s'agit alors d'un jeu à somme nulle.

Le deuxième problème de mise en application relevé par Bernard concerne la prévisibilité. Lors de la remise de leurs budgets, les gouvernements provinciaux établissent les prévisions de revenus pour les années subséquentes. Cette démarche peut s'avérer dangereuse lorsqu'une part importante de ces revenus peut fluctuer sans que le gouvernement provincial ait de pouvoir sur la situation, ce qui est le cas pour la péréquation. Comme traité précédemment, l'importance des paiements peut changer rapidement à cause de la conjoncture économique. De plus, un changement de gouvernement peut amener des modifications majeures au niveau des règles de calcul, ce qui peut influencer les montants à recevoir.

Le troisième problème est la conséquence que peuvent avoir les paiements de péréquation sur les décisions économiques respectives de chacune des provinces ou bien comme le nomme Bernard, l'incitation pour les provinces bénéficiaires. Il a été expliqué plus haut que pour calculer la capacité fiscale de chaque province, c'est le taux moyen de taxation canadien qui est pris en compte et non le taux réel. Autrement, il serait facile pour une province d'abaisser son taux de taxation et ainsi recevoir plus de péréquation. L’impossibilité de pouvoir agir ainsi n'empêche cependant pas une province de prendre en compte l'effet possible des autres politiques économiques sur sa capacité à tirer des revenus des différentes assiettes fiscales. Ce type de comportement vise à obtenir une hausse de son paiement de péréquation. Par exemple, Smart (2007) a démontré que les provinces bénéficiaires ont des taux moyens de taxation plus élevés que les autres. Pourtant, les gouvernements savent qu'au niveau macro-économique, des hausses de taxes font fuir les activités économiques vers des provinces ayant un régime fiscal plus avantageux. Néanmoins, ils les mettent en application, car les pertes engendrées par la délocalisation de certaines sources de revenus seront compensées par une hausse du versement de la 
péréquation. Cela est possible puisqu'au moment de calculer cette dernière, un taux de taxation supérieur à la moyenne est rabaissé au niveau de celle-ci. En d'autres mots, une hausse de taxe d'un gouvernement provincial lui octroie plus de revenus, mais l'augmentation des taxes n'entraîne pas une hausse de revenu équivalent à la réalité, puisqu'il y a toujours le phénomène de fuite fiscale. La péréquation vient compenser cette perte des provinces qui taxent plus que les autres.

La Suisse a déjà connu de tels problèmes. Certains cantons n'étaient pas compétitifs avec les autres en matière fiscale, mais ils ne s'en souciaient guère. En effet, le manque à gagner de ne pas attirer de nouveaux contribuables ou investisseurs était compensé par les paiements de péréquation émis par le fédéral et les autres cantons (à la différence des provinces canadiennes, les États fédérés suisses contribuent au paiement de la péréquation). La population a néanmoins approuvé une réforme, qui est entrée en vigueur en 2008, pour changer le système de péréquation pour que ce type de comportement ne soit plus possible (Kirchgässner et Guptara, 2006 p. 34-35).

Pour revenir au Canada, il est à noter que les agissements économiques vont parfois à l'encontre d'une stratégie visant à recevoir plus de péréquation. Un contre-exemple intéressant est présenté par Bernard et Mabrouk (2010), c'est celui du gouvernement du Québec et d'Hydro-Québec. En 2006, le gouvernement a décidé de financer le Fonds des générations pour diminuer la dette de la province. Pour ce faire, la société d'État a commencé à verser des redevances à la province et en 2008-2009, le gouvernement a aussi augmenté le taux de dividende qu'Hydro-Québec verse de $50 \%$ à $75 \%$. Ces deux mesures combinées ont eu comme résultat de transférer 1,15 milliard dans les coffres du gouvernement $\mathrm{du}$ Québec. Ces mesures affectent la norme nationale du calcul de péréquation, augmentent l'assiette fiscale du Québec et, par conséquent, privent la province de 437 millions en paiement de péréquation. Cette politique surprend les deux auteurs: "Ce geste volontaire du Québec, qui l'amène à recevoir moins d'argent du gouvernement fédéral, peut paraître surprenant, d'autant plus que l'on sait qu'en général le Québec défend bec et ongles ses droits de péréquation ».

Le quatrième problème soulevé par Bernard a déjà été mentionné précédemment. Il s'agit du pourcentage de prise en compte des ressources naturelles dans le calcul de la péréquation. Certains soutiennent que les ressources appartiennent aux provinces et que 
par conséquent celles-ci devraient être les seules à en profiter. D'autres soutiennent plutôt que ces revenus servent à offrir des services et qu'ils doivent être utilisés pour assurer une qualité de service semblable à un taux de taxation comparable partout au pays. En plus des problèmes soulevés par Bernard, il en existe bien d'autres.

Notamment, en ce qui concerne la péréquation, on peut aussi se demander à qui elle profite réellement. En effet, les transferts vont dans les coffres des provinces et non pas dans les poches des individus (Stevenson, 2004, p. 142). À l'intérieur du pays, les disparités de salaires continuent à être importantes selon le lieu de résidence. En effet, en 2013, le salaire d'une famille moyenne du Nouveau-Brunswick était de 67,340 \$ comparativement à 97,390 \$ pour l'Alberta (statistiques Canada). L'objectif d'offrir une qualité de service comparable partout au pays ne signifie aucunement d'égaliser le salaire des individus ou de leur assurer une même qualité de vie d'un point de vue monétaire. Ainsi, étant motivés par l'appât de salaire plus élevé, plusieurs résidents du pays ont migré vers l'Ouest. L'Alberta et la Colombie-Britannique ont accueilli la grande partie des migrants interprovinciaux avec respectivement 483,000 et 456,000, depuis 1976-1977. Sur la même période et pour la même catégorie d'immigration, le Québec a eu un solde négatif de 465,000 individus (statistiques Canada). Ainsi, on peut croire qu'il y a un lien à faire entre le salaire moyen et la migration. Accorder une qualité de service semblable ne suffirait donc pas à inciter la population à demeurer dans sa province natale.

Un autre problème soulevé est que la péréquation ne tient pas compte du coût de la vie qui peut être sensiblement différent d'une province à l'autre. Les assiettes fiscales sont calculées en fonction des revenus et non en fonction du pouvoir d'achat. Cette situation est surtout avantageuse pour le Québec puisque le coût de la vie est de 8,5\% moins élevé que dans les autres provinces bénéficiaires. La formule fédérale actuelle sous-estime donc la richesse réelle par habitant. Il y a un autre facteur qui n'est pas pris en compte dans le calcul des paiements et qui avantage le Québec. C'est que les travailleurs œuvrent volontairement moins que dans les autres provinces (de l'ordre de $4 \%$ ) pour profiter de plus de temps libre. Cela a comme conséquence de diminuer la capacité fiscale du gouvernement québécois. Ainsi, indirectement la péréquation paie pour les heures de loisir que les Québécois s'offrent et qui n'a pas son pendant dans les autres provinces (Fortin, 2014). Les 
problèmes qui viennent d'être décrits sont surtout dus à la structure du programme. De ceux־ci découlent un autre type de complication, qui est plutôt d'ordre politique.

\section{Problèmes politiques découlant de la péréquation}

L’objectif de la péréquation qui est d'assurer une qualité de service équivalent à un taux de taxation comparable n'est pas réellement remis en doute par les provinces ou leur population. Il en va autrement pour d'autres éléments. L'exemple évoqué précédemment de la prise en compte (ou pas) des ressources dans le calcul en est un bon exemple. L'Accord de l'Atlantique signé en 1985 qui excluait les ressources naturelles se situant au large de la Nouvelle-Écosse et de Terre-Neuve du calcul de la péréquation devait être renégocié en 2004. Au début des négociations, Paul Martin souhaitait que les revenus des ressources de ces deux provinces soient de nouveau pris en compte. Le premier ministre de Terre-Neuve à l'époque, Danny Williams, était complètement en désaccord avec cette idée. Ce type de chicane fédéral/provincial dont seul le Canada a le secret fit les manchettes lorsque Williams décida le 23 décembre 2004 de retirer les drapeaux canadiens des édifices provinciaux de Terre-Neuve. Finalement, Paul Martin accepta de continuer à exempter à $100 \%$ les ressources des deux provinces, mais principalement pour des raisons électoralistes puisque le scandale des commandites venait d'éclater et que chaque comté allait avoir une importance primordiale (Higgins, 2012).

Ce problème allait cependant revenir sur le devant de la scène avec l'élection du Parti conservateur de Stephen Harper. En effet, en 2007, ce dernier revoit la formule de péréquation et annule les accords passés avec le précédent gouvernement. Les ressources gazières et pétrolières des deux provinces seront alors prises en considération à la hauteur de $50 \%$. La colère de Williams est telle qu'il lance une campagne qu'il surnomme $A B C$ (Anything but Conservative) à l'élection fédérale suivante.

La péréquation continue de faire partie des discussions de la politique canadienne. Le sujet s'est d'ailleurs immiscé dans le débat concernant l'oléoduc Énergie Est. C'est le premier ministre de la Saskatchewan, Brad Wall, qui a évoqué le sujet après une conférence de presse de Denis Coderre contre le projet de TransCanada. Selon Wall, Québec devrait redonner à Ottawa les 10 milliards en péréquation qu'il reçoit par année s’il n'approuve pas l'oléoduc. Il mentionne que l'exportation des ressources de l'Ouest par ce 
pipeline permettrait de hausser les revenus de l'Alberta et de la Saskatchewan et que grâce au paiement de péréquation, ce sont tous les Canadiens qui en profiteraient. Selon certains (Fillion, 2016), ce genre de raisonnement doit être considéré comme un raccourci, car il évacue une série d'autres facteurs, comme le prix des ressources et les revenus fiscaux de chacune des provinces dans un contexte de ralentissement économique. Il est à noter que Brad Wall a fait sa déclaration durant la campagne électorale de sa province et comme Stevenson (2006) le fait remarquer: "A campaign around the issue of equalization is probably more likely to bear fruit than one on the more nebulous issue of vertical fiscal imbalance». Wall a d'ailleurs facilement été réélu, mais la controverse Énergie Est/péréquation risque de continuer à faire parler d'elle. Un observateur politique du Globe and Mail a d'ailleurs écrit ceci à propos de l'oléoduc et des conséquences financières qui pourraient s'y rapporter (ou pas) : « the straight line between Alberta's oil and the standard of living of every Canadian. [...] Will this one nation-building project ultimately tear us apart?»(Yakabuski, 2016).

Aussi, l'argument de la péréquation revient souvent dans l'éternel débat fédéraliste/souverainiste qui anime la population du Québec. La péréquation est souvent invoquée par les tenants de la première idée pour critiquer une éventuelle indépendance de la province. Toutefois, ceux qui souhaitent la séparation prétendent que la péréquation n'est pas vraiment avantageuse pour le Québec et qu'il vaudrait mieux garder tout l'argent que la province envoie à Ottawa. L'idée ici n'est pas de refaire ce débat ou d'essayer de le trancher, mais plutôt de donner un autre exemple de comment la péréquation influence les acteurs politiques.

Pierre-Elliott Trudeau voyait dans la péréquation un symbole d'unité et d'équité canadienne qui, selon lui, reflétait les valeurs canadiennes d'un océan à l'autre. Les exemples précédents démontrent que ce n'est pas toujours vrai et que l'importance accordée à l'équité et l'égalité peut varier selon les conjonctures et les acteurs. Selon Stevenson (2006), il demeure impossible qu'un consensus entre les provinces, ainsi qu'entre celles-ci et le gouvernement central, puisse survenir. En effet, les gouvernements n'ont rien à gagner à se dire satisfaits des ententes et ont tout à perdre à proclamer le contraire. Stevenson va même jusqu'à dire à propos de la péréquation: "Only the weather has been as durable a 
source of Canadian unhappiness, and even that may decline in importance with global warming ".

\section{Conclusion}

En résumé, la péréquation est un transfert fédéral qui vise à assurer des services publics de qualité comparable avec une fiscalité semblable aux provinces. Il s'agit d'un transfert inconditionnel. Depuis son application en 1957, la formule de calcul n'a cessé de changer pour s'adapter aux aléas économiques de la société canadienne. Tous les changements n'ont pas été en mesure de pallier aux nombreux problèmes de mise en application que le programme suscite. En plus de ceux-ci, la péréquation est une importante source de conflits entre le fédéral et les provinces, en plus de créer de l'animosité entre celles-ci. Néanmoins, malgré les problèmes et les querelles, la péréquation apparaît comme essentielle pour assurer une vitalité économique sensiblement pareille entre les provinces. On peut être porté à croire que de ne pas avoir ce type de programme accentuerait les différences entre les provinces. Encore plus que les vives discussions actuelles, cela pourrait remettre en cause l'essence même de l'union entre les dix provinces qui composent le Canada.

\section{Bibliographie}

ANDERSON, George. Fiscal Federalism : A Comparative Introduction, Oxford University Press, 2010, 104 pages.

BAKVIS, Herman et Grace SKOGSTAD. Canadian Federalism : Performance, Effectiveness, and Legitimacy, Oxford University Press, 2008, 488 pages.

BERNARD, Jean-Thomas et S.B. MABROUK. «Péréquation et comportement stratégique des provinces bénéficiaires : un contre-exemple intriguant », Canadian Public Policy, 36(2), 2010, p. 215 à 225.

BERNARD, Jean-Thomas. 2012. «Le programme canadien de péréquation : fondements, réalisations et défis », L'idée fédérale, [en ligne], http://ideefederale.ca/documents/Perequation.pdf, (3 avril 2016).

KIRCHGÄSSNER, Gebhard, et Prabhu GUPTARA. « Sustainable public finances with sub-federal fiscal autonomy: the case of Switzerland », dans BLINDENBACHER, Raoul et Abigail O. KAROS (sous la dir. De), Dialogues on the practice of fiscal Federalism. Comparative perspectives, 2006, Canada, Forum des fédérations, p. 34-35. 
COURCHENE. T.J., «Paiements de péréquation», Encyclopédie canadienne, [en ligne], 2013, http://www.encyclopediecanadienne.ca/fr/article/paiements-de-perequation/, (3 avril 2016)

DI MATTEO Livio. "A primer on equalization in Canada», Fraser Institute, [en ligne], 2015, https://www.fraserinstitute.org/blogs/primer-equalization-canada, (3 avril 2016).

FILLION, Gérald. «Péréquation : le Québec a-t-il une obligation morale d'approuver Énergie Est? », Radio-canada, [en ligne], 2016, http://blogues.radio-canada.ca/geraldfillion/2016/01/22/perequation-le-quebec-a-t-il-uneobligation-morale-de-dire-oui-a-energie-est/, (3 avril 2016).

FORTIN, Pierre. «Pourquoi le Québec reçoit-il des milliards en péréquation? », L'Actualité, [en ligne], 2014, http://www.lactualite.com/politique/pourquoi-le-quebec-recoit-il-des-milliards-en-perequation/, $\quad(3$ avril 2016).

GOUVERNEMENT DU CANADA. Loi constitutionnelle de 1982, [en ligne], http://laws-lois.justice.gc.ca/ fra/const/page-16.html\#h-53, (3 avril 2016).

HIGGINS, Jenny. "The 2005 Offshore Arrangement », Newfoundland and Labrador Heritage Web Site, [en ligne], 2012, http://www.heritage.nf.ca/articles/politics/offshore-arrangement-2005.php, (3 avril 2016).

MINISTÈRE DES FINANCES DU CANADA. "Soutien fédéral aux provinces et aux territoires », [en ligne], 2016, https://www.fin.gc.ca/fedprov/mtp-fra.asp, (3 avril 2016).

NADEAU, Jean-François. "Transferts fédéraux aux provinces et aux territoires en 2014-2015 », Bureau du Directeur parlementaire du budget, [en ligne], 2014, http://www.pbo-dpb.gc.ca/web/default/files/files/ files/TransferPayments_FR.pdf, (3 avril 2016).

ROY-CÉSAR, Édison. «La formule de péréquation du Canada », Publications de recherche de la bibliothèque du Parlement, [en ligne], 2013, http://www.bdp.parl.gc.ca/content/lop/ResearchPublications/2008-20-f.htm\#a9, (3 avril 2016).

SMART, Michael. «Raising Taxes through Equalization », Canadian Journal of Economics, 40(4), 2007, p. 1188 à 1212.

STEVENSON, Garth. Unfulfilled Union : Canadian Federalism and National Unity, Montréal, McGill-Queen's University Press, 2004, 352 pages.

STEVENSON, Garth. «Fiscal Federalism and the Burden of History », Institute of Intergovernmental Relations, [en ligne], 2006, http://www.queensu.ca/iigr/sites/webpublish.queensu.ca.iigrwww/files/files/WorkingPapers/ fiscalImb/Stevenson.pdf, (3 avril 2016).

STATISTIQUES CANADA. «Le Quotidien », [en ligne], http://www.statcan.gc.ca/start-debut-fra.html, (3 avril 2016).

YAKABUSKI, Konrad. «Energy East : The pipeline that could tear Canada apart », [en ligne], 25 janvier 2016, http://www.theglobeandmail.com/opinion/energy-east-the-pipeline-that-could-tear-canada-apart/ article28357800/, The Globe and Mail, (3 avril 2016). 


\section{April 14, 2017 FEDERALISM-E}

Annexe I

Tableau 1 : Soutien fédéral aux provinces et aux territoires (millions de dollars)

\begin{tabular}{|c|c|c|c|c|c|c|c|c|c|c|}
\hline & 2007- & 2008- & 2009- & $2010-$ & 2011- & 2012- & 2013- & $2014-$ & 2015- & 2016- \\
\hline & 2008 & 2009 & 2010 & 2011 & 2012 & 2013 & 2014 & 2015 & 2016 & 2017 \\
\hline Principaux transferts $^{1}$ & & & & & & & & & & \\
\hline Transfert canadien en matière de & 21 & 22 & 24 & 25 & 26 & 28 & 30 & 32 & 34 & 36 \\
\hline santé $^{2}$ & 729 & 768 & 476 & 672 & 952 & 569 & 283 & 113 & 026 & 068 \\
\hline & 7חת & 10 & 10 & 11 & 11 & 11 & 12 & 12 & 12 & 13 \\
\hline de programmes sociaux ${ }^{3}$ & & 552 & 857 & 179 & 514 & 859 & 215 & 582 & 959 & 348 \\
\hline Péréguation 4 & & 13 & 14 & 14 & 14 & 15 & 16 & 16 & 17 & 17 \\
\hline & 925 & 462 & 185 & 372 & 659 & 423 & 105 & 669 & 341 & 880 \\
\hline Paiements compensatoires & & & & & & & & & & \\
\hline $\begin{array}{c}\text { associés aux ressources } \\
\text { extracôtières }^{5}\end{array}$ & 563 & 663 & 645 & 869 & 787 & 443 & 350 & 196 & 125 & 49 \\
\hline $\begin{array}{c}\text { Formule de financement des } \\
\text { territoires }\end{array}$ & 2279 & 2313 & 2498 & 2664 & 2876 & 3111 & 3288 & 3469 & 3561 & 3603 \\
\hline $\begin{array}{c}\text { Protection sur les transferts } \\
\text { totaux }^{7}\end{array}$ & & & & 525 & 952 & 680 & 56 & & & \\
\hline Paiement additionnel $^{8}$ & & & 74 & & & & & & & \\
\hline & 47 & 49 & 52 & 55 & 57 & 60 & 62 & 65 & 68 & 70 \\
\hline & 102 & 758 & 736 & 281 & 739 & 085 & 297 & 029 & 013 & 948 \\
\hline Répartition par habitant (dollars) & 1434 & 1499 & 1570 & 1628 & 1683 & 1731 & 1774 & 1832 & 1899 & 1961 \\
\hline
\end{tabular}

Source : Ministère des finances du Canada: https://www.fin.gc.ca/fedprov/mtp-fra.asp 


\section{April 14, 2017 FEDERALISM-E}

Tableau 2 : Soutien fédéral au Québec (millions de dollars)

\begin{tabular}{|c|c|c|c|c|c|c|c|c|c|c|}
\hline & \multirow{3}{*}{\begin{tabular}{|l|}
$2007-$ \\
2008 \\
\end{tabular}} & \multirow{3}{*}{$\begin{array}{l}2008- \\
2009\end{array}$} & \multirow{3}{*}{\begin{tabular}{|l|}
$2009-$ \\
2010
\end{tabular}} & \multirow{3}{*}{$\begin{array}{l}2010- \\
2011\end{array}$} & \multirow{3}{*}{\begin{tabular}{|l|}
$2011-$ \\
2012 \\
\end{tabular}} & \multirow{3}{*}{\begin{tabular}{|l|}
$2012-$ \\
2013 \\
\end{tabular}} & \multirow{3}{*}{\begin{tabular}{|l|}
$2013-$ \\
2014 \\
\end{tabular}} & \multirow{3}{*}{\begin{tabular}{|l|}
$2014-$ \\
2015 \\
\end{tabular}} & \multirow{3}{*}{$\begin{array}{l}2015- \\
2016\end{array}$} & \multirow{3}{*}{$\begin{array}{l}2016- \\
2017\end{array}$} \\
\hline & & & & & & & & & & \\
\hline & & & & & & & & & & \\
\hline \multicolumn{11}{|l|}{$\begin{array}{l}\text { Principaux } \\
\text { transferts }\end{array}$} \\
\hline $\begin{array}{c}\text { Transfert canadien } \\
\text { en matière de } \\
\text { santé }^{1}\end{array}$ & 5246 & 5471 & 5799 & 6124 & 6445 & 6836 & 7239 & 7420 & 7844 & 8300 \\
\hline $\begin{array}{l}\text { Transfert canadien } \\
\text { en matière de } \\
\text { programmes } \\
\text { sociaux }\end{array}$ & 2215 & 2452 & 2520 & 2590 & 2664 & 2759 & 2834 & 2908 & 2987 & 3072 \\
\hline Péréquation & 7160 & 8028 & 8355 & 8552 & 7815 & 7391 & 7833 & 9286 & 9521 & 10030 \\
\hline $\begin{array}{l}\text { Protection sur les } \\
\text { transferts totaux }\end{array}$ & & & & & 369 & 362 & & & & \\
\hline $\begin{array}{c}\text { Total - Soutien } \\
\text { fédéral }\end{array}$ & 14622 & 15952 & 16673 & 17267 & 17292 & 17349 & 17907 & 19614 & 20352 & 21402 \\
\hline $\begin{array}{c}\text { Répartition par } \\
\text { habitant (dollars) }\end{array}$ & 1902 & 2057 & 2128 & 2180 & 2162 & 2148 & & & & \\
\hline
\end{tabular}

Source : Ministère des finances du Canada https://www.fin.gc.ca/fedprov/mtp-fra.asp 